\title{
Bayesian Semiparametric Modeling for Matched Case-Control Studies with Multiple Disease States
}

\author{
Samiran Sinha, Bhramar Mukherjee and Malay Ghosh* \\ Department of Statistics, University of Florida, Gainesville, Florida 32611, U.S.A. \\ *email: ghoshm@stat.ufl.edu
}

\begin{abstract}
Summary
We present a Bayesian approach to analyze matched "case-control" data with multiple disease states. The probability of disease development is described by a multinomial logistic regression model. The exposure distribution depends on the disease state and could vary across strata. In such a model, the number of stratum effect parameters grows in direct proportion to the sample size leading to inconsistent MLE's for the parameters of interest even when one uses a retrospective conditional likelihood. We adopt a semiparametric Bayesian framework instead, assuming a Dirichlet process prior with a mixing normal distribution on the distribution of the stratum effects. We also account for possible missingness in the exposure variable in our model. The actual estimation is carried out through a Markov chain Monte Carlo numerical integration scheme. The proposed methodology is illustrated through simulation and an example of a matched study on low birth-weight of newborns (Hosmer and Lemeshow, 2000) with two possible disease groups matched with a control group.
\end{abstract}

KEY WORDs: Conditional inference; Dirichlet mixture; Exponential family; Gibbs sampling. 


\section{Introduction}

Case-control studies have received a great deal of attention over the last few decades both from statisticians and epidemiologists. The analysis is based on the comparison of persons having a disease (the cases) with those not having the disease (the controls) and assess the effect of exposure variables on the probability of developing the disease. For efficient use of data, in such studies, one usually implements a matched design, matching one or more controls with a case on the basis of some prognostic factors such as age, family background etc. In some such situations it is natural to note that the disease state might have more than one category, i.e., we may have subdivisions within the "cases". For example, for patients diagnosed with cancer, they may have cancer of stage-I, stage-II or stage-III at the time of the diagnosis which is an example of ordinal disease categories. We may also notice nominal categories for disease states such as patients classified into having one eye or both eyes damaged. To our best knowledge there has been hardly any Bayesian and very little frequentist work for analyzing matched data when one has multiple disease states. Along with considering polytomous disease states we extend the typical methodology for analyzing case-control data in the following way. In analyzing a matched study one usually considers an appropriate conditional likelihood to eliminate the stratum effect parameters involved in determining the disease probability, while non-specific exposure distributions are assumed to satisfy Prentice-Pyke (1979) type constraints. In contrast, we assume the exposure distribution to be a member of the exponential family and allow for the exposure distribution to vary across strata. In such a situation, even the conditional likelihood involves some nuisance parameters which grow in direct proportion to sample size, giving rise to inconsistency of the conditional MLE's. We adopt a semiparametric Bayesian approach to circumvent this problem and estimate the parameters of interest through a numerical computation scheme. We also account for possible missingness in the exposure variable through modeling the distribution of the exposure variable. 
The example considered in the paper involves a matched case-control dataset coming from a low birth weight study conducted by the Baystate Medical Center in Springfield, Massachusetts. The dataset is discussed in Hosmer and Lemeshow (2000, Section 1.6.2) and is used as an illustrative example of analyzing a matched case-control study in Chapter 7 of their book. Low birth weight, defined as birth weight less than 2500 grams, is a cause of concern for a newborn as infant mortality and birth defect rates are very high for low birth weight babies. The data was matched according to the age of the mother. A woman's behavior during pregnancy (smoking habits, diet, prenatal care) can greatly alter the chances of carrying the baby to terms. The goal of the study was to determine whether these variables were "risk factors" in the clinical population served by Baystate Medical Center. Using the actual birth weight observations we divided the cases, namely, the low birth-weight babies into two categories, very low (weighing less than 2000 gms) and low (weighing between 2000 to $2500 \mathrm{gms}$ ) and tried to assess the impact of smoking habits of mother on the chance of falling in the two low birth-weight categories. Presence of uterine irritability in mother and mother's weight at last menstruation period were considered as relevant covariates. It was noted that smoking mothers had a higher relative risk of having a low birth weight child when compared to a non-smoking mother. However, the risk of having a very low birth weight child did not depend on smoking significantly. This observation could not be made without the classification of the data into multiple low birth weight groups, illustrating the relevance of such a type of analysis in certain situations.

In many real studies, one often does not have access to exposure information on all the subjects under study. In such situations, rather than ignoring the available partial information one gains in terms of estimation accuracy of the parameters of interest if the distribution of the missing exposure or the missingness process is stochastically modeled. Although the frequentist literature contains a number of papers for matched case control studies and treatment of missing covariate information (see e.g., Satten and Carroll, 2000; Paik and Sacco, 2000; Rathouz, Satten and Carroll, 2002), unified Bayesian methods addressing these prob- 
lems are needed. Zelen and Parker (1986), Nurminen and Mutanen (1987) and Ashby et al. (1993) considered case-control problems in a Bayesian formulation when the risk factor was a binary variable, the stratum effect was a constant, and there were no missing covariates. Müller and Roeder (1997) and Müller et al. (1999) considered a semiparametric model for unmatched case-control problems with continuous and possibly missing covariates, and binary disease status. Seaman and Richardson (2001) extended the Müller-Roeder approach for categorical covariates, and brought out the connection between the Zelen-Parker and Müller-Roeder approach in absence of measurement error. None of these papers considered a multicategory disease status in a matched case control set-up.

The present paper intends to develop an approach to case-control studies with a multicategory variable $\mathbf{D}$ denoting disease states, a completely observed covariate $\mathbf{Z}$, a vector of exposure variables or risk factors $\mathbf{X}$ which could potentially contain some missing observations. The exposure variables could be discrete or continuous and we assume that $\mathbf{X} \mid \mathbf{D}, \mathbf{Z}$ has a distribution coming from a exponential family which may have different natural parameters across strata. We will assume a Dirichlet process with a mixing normal distribution prior on the distribution of the stratum effects and normal priors on the other regression parameters and estimate all the parameters via a Markov Chain Monte Carlo computing scheme. This is a major departure from the usual frequentist as well as the Bayesian approach of assuming that the distribution of exposure variable is not affected by any stratum effect except through the measured covariates. This last assumption may not hold in many situations. For example, matching for cancer patients is often done from their family and smoking is a natural exposure to consider. The distribution of the exposure may depend on genetic traits in the family which may affect the disease distribution in different families in different ways and may not be measurable as a covariate. The present Bayesian approach will allow us to model stratum effects on the exposure distribution for highly stratified data and account for missing exposure information.

The outline of the remaining sections is as follows. In Section 2 we introduce notation and 
model assumptions. Section 3 contains the conditional likelihood and the priors. In Section 4 we analyze the low birth-weight data and discuss the MCMC computation scheme. Section 5 presents the results from a small simulation study comparing the proposed Bayesian semiparametric method with two possible parametric Bayesian alternatives. Section 6 contains concluding remarks. The Appendix contains details of some calculations and computation scheme.

Before concluding this section we highlight some of the new features of this article. First, ours seems to be the first attempt towards an analysis of matched case-control studies with multiple disease states. Second, the introduction of the semiparametric Bayesian method overcomes the difficulties associated with the conventional frequentist procedure when the number of nuisance parameters grows in direct proportion to the sample size even in the retrospective conditional likelihood. Third, we provide a unified analysis for both discrete and continuous exposure variables and account for possible missingness in exposure observations.

\section{Model and Notation}

Let $D_{i j}$ denote the disease state of the $j$-th individual in the $i$-th stratum $S_{i}$. Suppose that there are $(K+1)$ nominal levels of the disease variable, with $D_{i j}=k$ denoting disease state $k, k=1, . ., K$ and $D_{i j}=0$ denoting the control group. We assume that there is 1 case matched with $M$ controls in each stratum and we have $n$ strata in all. For ease of notation, we present our models with a single exposure $X$, but the model could easily be extended to the case when one has a set of independent multiple exposures each having a distribution coming from the exponential family. The disease probabilities for each of the $K$ groups are modeled through $K$ logits as in a multinomial logistic regression model:

$$
\log \frac{P\left[D_{i j}=k \mid S_{i}, \mathbf{Z}_{i j}, X_{i j}\right]}{P\left[D_{i j}=0 \mid S_{i}, \mathbf{Z}_{i j}, X_{i j}\right]}=\beta_{0 k}\left(S_{i}\right)+\boldsymbol{\beta}_{1 k}^{T} \mathbf{Z}_{i j}+\beta_{2 k} X_{i j} \quad \text { for } \quad k=1, \cdots, K .
$$

Each $\boldsymbol{\beta}_{1 k}$ is a $p \times 1$ column vector and $\mathbf{Z}_{i j}=\left(Z_{i j}^{(1)}, \cdots, Z_{i j}^{(p)}\right)^{T}$ is the vector of $p$ completely observed covariates. 
For our example with the low birth-weight data as described in Section 1, we have two disease states with $K=2$. Then $\exp \left(\beta_{21}\right)$ signifies the odds of having low birth weight baby for a mother who smokes relative to one who does not smoke and similarly $\exp \left(\beta_{22}\right)$ is the risk of a very low birth weight child for a mother who smokes relative to one who does not. The conditional probabilities of the disease variable given the covariate, exposure and the stratum are given by,

$$
P\left(D_{i j}=k \mid S_{i}, \mathbf{Z}_{i j}, X_{i j}\right)=\frac{\exp \left\{\beta_{0 k}\left(S_{i}\right)+\boldsymbol{\beta}_{1 k}^{T} \mathbf{Z}_{i j}+\beta_{2 k} X_{i j}\right\}}{1+\sum_{r=1}^{K} \exp \left\{\beta_{0 r}\left(S_{i}\right)+\boldsymbol{\beta}_{1 r}^{T} \mathbf{Z}_{i j}+\beta_{2 r} X_{i j}\right\}} \text { for } k=1, \cdots, K \text {. }
$$

and,

$$
P\left(D_{i j}=0 \mid S_{i}, \mathbf{Z}_{i j}, X_{i j}\right)=\frac{1}{1+\sum_{r=1}^{K} \exp \left\{\beta_{0 r}\left(S_{i}\right)+\boldsymbol{\beta}_{1 r}^{T} \mathbf{Z}_{i j}+\beta_{2 r} X_{i j}\right\}} .
$$

To cover both discrete as well as continuous exposures, we assume a general exponential family of distributions for the exposure variable in the control population with respect to some finite dominating measure $\mu$, i.e.,

$$
f\left(X_{i j} \mid S_{i}, \mathbf{Z}_{i j}, D_{i j}=0\right)=\exp \left[\xi_{i j}\left\{\theta_{i j} X_{i j}-b\left(\theta_{i j}\right)\right\}+c\left(\xi_{i j}, X_{i j}\right)\right]
$$

The natural parameters are modeled as a regression function of the completely observed covariates, namely, $\theta_{i j}=\gamma_{0 i}+\gamma_{1}^{T} \mathbf{Z}_{i j}$, where $\gamma_{1}^{T}=\left(\gamma_{11}, \cdots, \gamma_{1 p}\right)$. The dependence of the exposure distribution on the stratum is captured through the varying intercepts $\gamma_{0 i}$.

\section{Likelihood, Priors and Posteriors}

Before writing out the conditional likelihood we need some technical results stated in the following as lemmas. These lemmas follow by repeating essentially the proofs of Lemmas 1-3 of Sinha et al. (2003). The details are omitted.

LEMMA 1. Under assumptions (2)-(4) the distribution of the exposure variable in a given disease state $k$, namely, $f\left(X_{i j} \mid S_{i}, \mathbf{Z}_{i j}, D_{i j}=k\right)$ is also of general exponential form with scale 
parameter $\xi_{i j}$ and natural parameter $\theta_{i j k}^{*}=\theta_{i j}+\xi_{i j}^{-1} \beta_{2 k}$ for $k=1, \cdots, K$.

LEMMA 2. Under the same set of assumptions,

$$
\frac{P\left(D_{i j}=k \mid S_{i}, \mathbf{Z}_{i j}\right)}{P\left(D_{i j}=0 \mid S_{i}, \mathbf{Z}_{i j}\right)}=\exp \left\{\beta_{0 k}\left(S_{i}\right)+\boldsymbol{\beta}_{1 k}^{T} \mathbf{Z}_{i j}\right\} \times \exp \left[\xi_{i j}\left\{b\left(\theta_{i j k}^{*}\right)-b\left(\theta_{i j}\right)\right\}\right] .
$$

We need one more Lemma to write out the conditional likelihood.

LEMMA 3.

$$
\begin{aligned}
& \frac{P\left(D_{i s}=k \mid S_{i}, \mathbf{Z}_{i s}\right) / P\left(D_{i s}=0 \mid S_{i}, \mathbf{Z}_{i s}\right)}{\sum_{j=1}^{M+1} P\left(D_{i j}=k \mid S_{i}, \mathbf{Z}_{i j}\right) / P\left(D_{i j}=0 \mid S_{i}, \mathbf{Z}_{i j}\right)} \\
= & \frac{\exp \left(\boldsymbol{\beta}_{1 k}^{T} \mathbf{Z}_{i s}\right) \exp \left[\xi_{i s}\left\{b\left(\theta_{i s k}^{*}\right)-b\left(\theta_{i s}\right)\right\}\right]}{\sum_{j=1}^{M+1} \exp \left(\boldsymbol{\beta}_{1 k}^{T} \mathbf{Z}_{i j}\right) \exp \left[\xi_{i s}\left\{b\left(\theta_{i j k}^{*}\right)-b\left(\theta_{i j}\right)\right\}\right]} \text { for } s=1, \cdots, M+1, i=1, \cdots, n .
\end{aligned}
$$

Without loss of generality we may assume that the first subject in each stratum is a case and if we denote the disease state (type of case) in stratum $i$ as $k_{i}$ ( $k_{i}$ could assume any of the values $1, . ., K)$, then the conditional likelihood given that there is one case in each stratum will be of the form:

$$
\begin{aligned}
L_{c} & \propto \prod_{i=1}^{n} P\left\{D_{i 1}=k_{i}, D_{i j}=0(j=2, \cdots, M+1), X_{i j}(j=1, \cdots, M+1) \mid S_{i}, \mathbf{Z}_{i j}, \sum_{j=1}^{M+1} D_{i j}=k_{i}\right\} \\
& =\prod_{i=1}^{n}\left\{f\left(X_{i 1} \mid S_{i}, \mathbf{Z}_{i 1}, D_{i 1}=k_{i}\right) \prod_{j=2}^{M+1} f\left(X_{i j} \mid S_{i}, \mathbf{Z}_{i j}, D_{i j}=0\right)\right\} \\
& \times \prod_{i=1}^{n} \frac{P\left(D_{i 1}=k_{i} \mid S_{i}, \mathbf{Z}_{i 1}\right) \prod_{j=2}^{M+1} P\left(D_{i j}=0 \mid S_{i}, \mathbf{Z}_{i j}\right)}{\sum_{l=1}^{M+1} P\left(D_{i l}=k_{i} \mid S_{i}, \mathbf{Z}_{i l}\right) \prod_{j \neq l}^{M+1} P\left(D_{i j}=0 \mid S_{i}, \mathbf{Z}_{i j}\right)} \\
& =\prod_{i=1}^{n+1}\left\{f\left(X_{i 1} \mid S_{i}, \mathbf{Z}_{i 1}, D_{i 1}=k_{i}\right) \prod_{j=2}^{M+1} f\left(X_{i j} \mid S_{i}, \mathbf{Z}_{i j}, D_{i j}=0\right)\right\} \\
& \times \prod_{i=1}^{n} \frac{P\left(D_{i 1}=k_{i} \mid S_{i}, \mathbf{Z}_{i 1}\right) / P\left(D_{i 1}=0 \mid S_{i}, \mathbf{Z}_{i 1}\right)}{\sum_{j=1}^{M+1} P\left(D_{i j}=k_{i} \mid S_{i}, \mathbf{Z}_{i j}\right) / P\left(D_{i j}=0 \mid S_{i}, \mathbf{Z}_{i j}\right)}
\end{aligned}
$$

In many situations one may not have all observations recorded on the exposure variable. For example in the famous endometrial cancer dataset as discussed in Breslow and Day 
(1980), 16\% observations are missing on a possible risk factor obesity. In such situations, a typical conditional frequentist matched analysis loses the entire information on a subject with a single missing exposure. Modeling the exposure distribution in such situations leads to more efficient estimation of parameters of interest as compared to completely ignoring the partial information that is still available (Satten and Kupper, 1993a, 1993b; Satten and Carroll, 2000; Sinha et al., 2003).

We modify the above likelihood appropriately for the situations when the exposure variable may contain some missingness. Let $\delta_{i j}$ be an indicator variable for missing exposures defined in the following manner:

$$
\delta_{i j}= \begin{cases}1 & \text { if } X_{i j} \text { is observed, } \\ 0 & \text { if } X_{i j} \text { is missing, }, i=1, \cdots, n \text { and } j=1, \cdots, M+1\end{cases}
$$

We also assume that the distribution of $\delta_{i j}$ does not involve the parameters $\boldsymbol{\beta}_{1 k}, \beta_{2 k}, \boldsymbol{\gamma}_{1}$ and $\gamma_{0}^{T}=\left(\gamma_{01}, \cdots, \gamma_{0 n}\right)$. The conditional likelihood including missingness of the exposure variable is seen to be,

$$
\begin{aligned}
L_{c} & \propto \prod_{i=1}^{n}\left\{f\left(X_{i 1} \mid S_{i}, \mathbf{Z}_{i 1}, D_{i 1}=k_{i}\right)^{\delta_{i 1}} \prod_{j=2}^{M+1} f\left(X_{i j} \mid S_{i}, \mathbf{Z}_{i j}, D_{i j}=0\right)^{\delta_{i j}}\right\} \\
& \times \prod_{i=1}^{n} \frac{P\left(D_{i 1}=k_{i} \mid S_{i}, \mathbf{Z}_{i 1}\right) / P\left(D_{i 1}=0 \mid S_{i}, \mathbf{Z}_{i 1}\right)}{\sum_{j=1}^{M+1} P\left(D_{i j}=k_{i} \mid S_{i}, \mathbf{Z}_{i j}\right) / P\left(D_{i j}=0 \mid S_{i}, \mathbf{Z}_{i j}\right)} .
\end{aligned}
$$

This likelihood involves the parameters $\boldsymbol{\beta}_{1}^{p \times K}=\left(\boldsymbol{\beta}_{11}, \cdots, \boldsymbol{\beta}_{1 K}\right), \boldsymbol{\beta}_{2}^{T}=\left(\beta_{21}, \cdots, \beta_{2 K}\right), \boldsymbol{\gamma}_{1}$ and $\gamma_{0}$

We consider mutually independent normal priors: $\boldsymbol{\beta}_{1 k} \sim N_{p}\left(\boldsymbol{\mu}_{\beta_{1 k}}, \sigma_{\beta_{1 k}}^{2} \boldsymbol{I}_{p}\right)$ for $k=1, \cdots, K$. $\boldsymbol{\beta}_{2} \sim N_{K}\left(\boldsymbol{\mu}_{\beta_{2}}, \sigma_{\beta_{2}}^{2} \boldsymbol{I}_{K}\right) \gamma_{1} \sim N_{p}\left(\boldsymbol{\mu}_{\gamma_{1}}, \sigma_{\gamma_{1}}^{2} \boldsymbol{I}_{p}\right)$

One of the key features of our approach is to retain the nuisance parameters $\gamma_{0 i}$ in the model and to put a Dirichlet with normal mixture prior on them, i.e., $\gamma_{0 i} \mid G \stackrel{i i d}{\sim} G$ where $G \sim D P\left(\alpha G_{0}\right)$ and $G_{0}$ is $N\left(\zeta_{0}, \sigma_{0}^{2}\right)$. Using the classical result of Antoniak (1974), it follows 
that

$$
\gamma_{0 i} \mid \gamma_{0 k}(k \neq i) \sim \frac{\alpha}{\alpha+n-1} N\left(\zeta_{0}, \sigma_{0}^{2}\right)+\frac{1}{\alpha+n-1} \sum_{k=1, k \neq i}^{n} I_{\gamma_{0 k}}\left(\gamma_{0 i}\right),
$$

where $I$ is the indicator function. The result allows the possibility of equal values of some $\gamma_{0 i}$ as well. As we will notice in our simulations, the fact that it can allow for equal values of $\gamma_{0 i}$ plays an important role in making the procedure robust over a wide spectrum of scenarios, from widely varying $\gamma_{0 i}$ 's to the case when they are all equal.

For our data analysis and simulations we compared our Bayesian semiparametric (BSP) method with two possible parametric Bayesian alternatives. The first one is a parametric Bayesian analogue of the method proposed by Satten and Carroll (2000) for matched case control studies with a single disease state. Satten and Carroll (2000) assumed a constant stratum effect on the exposure distribution, i.e., $\gamma_{0 i} \equiv \gamma_{0}$. In the parametric Bayesian analogue of their method (denoted by PBC, C standing for constant stratum effect) in the context of multiple disease states, we consider a normal distribution as a prior on this common stratum effect parameter $\gamma_{0}$ and carry out Bayesian analysis. The other parametric Bayesian alternative (denoted by PBV, V standing for varying stratum effects) allows for possibly varying $\gamma_{0 i}$ and assumes i.i.d. normal prior on each $\gamma_{0 i}$.

Remark 1. It follows from (9) that for very large values of $\alpha$ the BSP method is equivalent to the PBV method whereas for very small values of $\alpha$ it amounts to assuming a completely discrete prior on $\gamma_{0 i}$. In our numerical work we assumed a Gamma prior on $\alpha$ and resampled from the full conditional distribution of $\alpha$ using a latent beta variable as prescribed in Escobar and West (1995).

Remark 2. The entire analysis carries through if each stratum contains varying number of controls, with equations (5)-(9) remaining essentially the same with $M$ replaced by $M_{i}$ in the $i$-th stratum.

The estimation of the parameters is done by the Markov chain Monte Carlo numerical integration scheme. To generate random numbers from the posterior distributions of the pa- 
rameters we use a componentwise Metropolis Hastings scheme. We describe the computation scheme along with the analysis of the low birth-weight study in the following section.

\section{Example and Computing scheme}

In the previous sections we discussed the general methodology which we now apply to the matched case-control study for low birth weight data as described in Section 1. The matched data contain 29 strata and each stratum has one case and 3 controls. We denote the low birth weight and the very low birth weight group as disease state 1 and 2 respectively. One can possibly think of many different models for explaining the disease in terms of the possible covariates recorded in the data set. We consider smoking status of mother as a single exposure variable. Two other covariates, a binary variable denoting presence of uterine irritability (UI) in mother and weight of the mother at last menstrual period (LWT) are also included in the model.

As a starting point, we separated the 29 strata into two groups depending on whether the case belonged to low birth weight category 1 or 2 . We formed two cross-classification tables of birth weight category versus smoking status of mother during pregnancy period for these two separate matched samples and noted that, $\widehat{\mathrm{OR}(1}, 0)=3.4$ and $\widehat{\mathrm{OR}(2}, 0)=1.917$, where $\widehat{O R} \widehat{(k}, 0)$ denotes the odds ratio of maternal smoking habits for birth weight group $=k$ versus normal birth weight group $=0$. The two odds ratios demonstrate that the odds of having a low birth weight baby for a smoking mother as opposed to a non-smoking mother is higher in category 1 whereas for category 2 we notice a relatively weaker behavior in the same direction. The difference in the odds ratios in these two tables led us to use this data as a testbed example to illustrate our methods.

For our proposed analysis we have a stochastic distribution on the exposure variable which belongs to the exponential family. The binary variable smoking status is assumed to 
follow a Bernoulli distribution:

$$
f\left(X_{i j} \mid D_{i j}=0, \mathbf{Z}_{i j}, S_{i}\right)=p_{i j}^{X_{i j}}\left(1-p_{i j}\right)^{1-X_{i j}}
$$

so here $\xi_{i j}=1, \theta_{i j}=\ln \left(\frac{p_{i j}}{1-p_{i j}}\right), b\left(\theta_{i j}\right)=\ln \left(1+\exp \left(\theta_{i j}\right)\right)$ and $c\left(X_{i j}, \xi_{i j}\right)=0$. Also here $K=2$, $p=2, n=29$ and $M=3$. Using Lemmas 1,2 and 3 we obtain the conditional likelihood for the whole data:

$$
\begin{aligned}
L_{c} & \propto \prod_{i=1}^{n}\left\{\exp \left\{\theta_{i 1}^{*} X_{i 1}-\ln \left(1+\exp \left(\theta_{i 1}^{*}\right)\right)\right\}\right. \\
& \times \exp \left[\sum_{j=2}^{M+1}\left\{\theta_{i j} X_{i j}-\ln \left(1+\exp \left(\theta_{i j}\right)\right)\right\}\right] \\
& \left.\times \frac{\exp \left(\boldsymbol{h}_{i}^{T} \boldsymbol{\beta}_{11} Z_{i 1}^{(1)}+\boldsymbol{h}_{i}^{T} \boldsymbol{\beta}_{12} Z_{i 1}^{(2)}\right) \times \frac{\left(1+\exp \left(\theta_{i 1}^{*}\right)\right)}{\left(1+\exp \left(\theta_{i 1}\right)\right)}}{\sum_{j=1}^{M+1} \exp \left(\boldsymbol{h}_{i}^{T} \boldsymbol{\beta}_{11} Z_{i j}^{(1)}+\boldsymbol{h}_{i}^{T} \boldsymbol{\beta}_{12} Z_{i j}^{(2)}\right) \times \frac{\left(1+\exp \left(\theta_{i j}^{*}\right)\right)}{\left(1+\exp \left(\theta_{i j}\right)\right)}}\right\}
\end{aligned}
$$

where $\theta_{i j}^{*}=\theta_{i j}+\boldsymbol{h}_{i}^{T} \boldsymbol{\beta}_{2}$. Since the value of $k$ (i.e., disease type) is completely determined by knowing the stratum we omit the subscript $k$ for $\theta_{i j k}^{*}$ in the above expression; $Z_{i s}^{(1)}$ and $Z_{i s}^{(2)}$ denote the observed value of the two covariates UI and LWT for the $s$-th subject in the $i$-th stratum respectively and $\boldsymbol{h}_{i}$ is defined as $\boldsymbol{h}_{i}=\left(h_{i 1}, \cdots, h_{i K}\right)^{T}$ where,

$$
h_{i r}=\left\{\begin{array}{l}
1 \quad \text { if } D_{i 1}=r \\
0 \quad \text { otherwise }, i=1, \cdots, n \text { and } r=1, \cdots, K
\end{array}\right.
$$

Our analysis is based on normal priors centered at zero with large variances for all the regression parameters. In instances (such as ours) when prior elicitation is not possible, these priors usually lead to posteriors relying more heavily on the data and protect against model failures. In many real applications, the practitioner may have a more precise knowledge about the sign and magnitude of the relative risk parameters and can suitably change the prior if necessary. We conducted a sensitivity analysis with several choices of prior parameters. For the regression parameters and the mixing normal distribution of the Dirichlet process we experimented with normal priors centered at zero and with variances 2, 4, 5, 6 and 9 . We used a Gamma prior on the mixing parameter $\alpha$ of the Dirichlet process and ran our analysis with both shape and size parameter set at $0.5,1,2,4,10,40,100,200$ and with many other 
possible pairs like $\mathrm{G}(0.5,4), \mathrm{G}(1,10), \mathrm{G}(10,40), \mathrm{G}(100,40), \mathrm{G}(200,10)$. We noted that the ultimate numerical estimates are reasonably stable over a varying range of prior parameters.

The results are reported for independent $\mathrm{N}(0,5)$ prior on each component of $\boldsymbol{\beta}_{11}, \boldsymbol{\beta}_{12}$ and $\boldsymbol{\beta}_{2}, \mathrm{~N}(0,6)$ as the mixing normal distribution for the Dirichlet process prior and a $\operatorname{Gamma}(2,2)$ prior for the mixing parameter $\alpha$.

We used componentwise Metropolis Hastings algorithm to generate random numbers from the full conditionals of the parameters. For generating observations from the posterior distribution of $\gamma_{0 i}, i=1, \ldots, n$, we used an algorithm proposed by Neal (2000) for simulating observations from posteriors of Dirichlet mixtures for non-conjugate cases. The details of the computation scheme are given in Sinha et al. (2003). The full conditional distributions for the parameters are presented in the appendix. We ran the chain typically from 7000-10000 iterations and calculated the diagnostic proposed by Gelman and Rubin (1992) as a measure of convergence.

Table 1 contains the posterior means, posterior standard deviations and 95\% HPD credible intervals for the parameters of interest under the proposed Bayesian Semiparametric method (BSP) and the parametric Bayes (PBC and PBV) methods as discussed before. In the parametric Bayes methods we used a $\mathrm{N}(0,6)$ prior on the constant $\gamma_{0}$ (in PBC) and i.i.d. $\mathrm{N}(0,6)$ prior on varying $\gamma_{0 i}$ 's (in PBV). To illustrate the methods in presence of missingness, we deleted $40 \%$ of exposure values completely at random (in the sense of Little and Rubin, 1987) and reran the three analyses. The results are presented in Table 2.

The full data analysis indicates that smoking of mother is a significant risk factor for low birth-weight category (category 1) and is not very significant in the very low birthweight category (category 2). UI on the other hand shows an opposite association, showing significance in category 2 and almost no significance in category 1 . LWT does not seem to be a significant covariate in any of the categories. The BSP and the PBV methods are in closer agreement whereas the PBC estimates show some numerical differences. 
Figure 1 shows a plot of the variance of the 29 stratum effects in the last 3000 MCMC samples. The average variance is approximately 2.3 showing that there indeed exists variability in the stratum effects. As a result the BSP and PBV methods which account for this variability are in close agreement, whereas the PBC method assuming constant stratum effect differs numerically from these two methods.

For the analysis with $40 \%$ missing observations on smoking one notices that the estimates corresponding to smoking in the BSP method comes closer to their full data counterparts even though the inferences are same in all three methods. As one might expect, with $40 \%$ missingness, the parameter estimates for smoking lose precision and the effect of smoking now appears to be not significant in both categories 1 and 2. Inferences on the other two covariates remain essentially unchanged when compared to full data inferences.

We also analyzed the data after collapsing category 1 and category 2 into only one category (birthweight less than 2500 gms). We carried out the Bayesian analysis for a simple matched case-control data (Sinha et al., 2003) and the usual conditional logistic regression (CLR) analysis (Breslow and Day, 1980). Table 3 shows that both BSP and PBV methods assuming varying stratum effects bring out the effect of mother's smoking on having low birth weight newborns and produce very similar results. The PBC and the CLR methods, assuming constant stratum effect are in closer agreement with each other and they do demonstrate the effect of smoking but not as precisely as the other two methods which allow varying stratum effects. Figure 1 again demonstrates the differences in results between these two classes of models. Obviously, without the finer classification into two weight categories, the fact that smoking is not so significant for category 2 and UI is appreciably significant for category 2 cannot be concluded from looking at the overall analysis. Thus the multicategory analysis may render some useful additional information to the practitioner. 


\section{Simulation Study}

In the low birth-weight data we noticed appreciable variability in the stratum effects. In practice, the experimenter may not have a prior idea about the nature of variability among stratum effects and there could be situations where the standard model assumption of constant stratum effect, i.e. $\gamma_{0 i} \equiv \gamma_{0}$ hold. Sinha et al. (2003) contain an example from equine epidemiology where the stratum effects have very small variability. We conducted a simulation study to ascertain the robustness of the BSP method even when variability in the stratum effects is negligible.

In order to simulate a realistic dataset for comparing the BSP, PBC and PBV methods, we decided to use the Low Birth Weight data itself. We generated a hypothetical 1:1 matched data set with 50 strata with one binary exposure variable (corresponding to $X$, smoking status of mother) and one binary covariate (corresponding to $Z$, presence of uterine irritability). The true values for $\beta_{11}, \beta_{12}, \beta_{21}$ and $\beta_{22}$ are chosen to be $0.20,1.80,1.40$ and 0.4 respectively, close to the estimates obtained by analyzing the low birth-weight data by the BSP method as presented in Table 1.

In order to elicit values for $\gamma_{1}$, the coefficient of $Z$ in the natural parameter of the exposure distribution, namely $\theta_{i j}$ on $Z$ we ran a logistic regression of $\mathrm{X}(\mathrm{SMOKE})$ on $\mathrm{Z}(\mathrm{UI})$ using the control sample and the fitted model turned out to be $\operatorname{logit}\left(\operatorname{pr}\left(X_{i j}\right)\right)=-0.734+0.579 Z_{i j}$. Accordingly, in the simulation study we used $\gamma_{1}=0.60$.

We followed the structure of our models as described before for simulating $Z, X$ and the trinomial variable $D$, indicating the birth weight category. For the low birth weight data occurrence of uterine irritability was reported in approximately $20 \%$ of the patients. Thus the completely observed covariate $\mathrm{Z}$ was generated first as a Bernoulli variable with

success probability 0.20 . Second, we generated the trinomial disease variable $D$. For the $i^{\text {th }}$ 
stratum, one should note that

$$
\operatorname{Pr}\left(D_{i 1}=0 \mid Z_{i 1}, Z_{i 2}, D_{i 1}+D_{i 2}=1 \text { or } 2, S_{i}\right)=\frac{1}{1+\frac{Q_{i 1}}{Q_{i 2}}}
$$

where, for $j=1,2$,

$$
Q_{i j}=\exp \left(\beta_{11} Z_{i j}\right) \frac{1+\exp \left(\theta_{i j}+\beta_{21}\right)}{1+\exp \left(\theta_{i j}\right)}+\exp \left(\beta_{12} Z_{i j}\right) \frac{1+\exp \left(\theta_{i j}+\beta_{22}\right)}{1+\exp \left(\theta_{i j}\right)}
$$

We generated a Bernoulli random variable with the above success probability and if this variable assumed a value 1 , the simulated value for $D_{i 1}$ was taken to be 0 implying that the first subject in the $i$-th stratum is a member of the control population. Let, for $k=1,2$,

$$
p_{i k}^{*}=\frac{1}{1+\exp \left[z_{i k}\left(\beta_{12}-\beta_{11}\right)\right] \frac{1+\exp \left(\theta_{i k}+\beta_{22}\right)}{1+\exp \left(\theta_{i k}+\beta_{21}\right)}} .
$$

If $D_{i 1}=0$, we determine $D_{i 2}$ according to a Bernoulli draw with success probability $p_{i 2}^{*}$; set $D_{i 2}=1$ if it results in a success otherwise set $D_{i 2}=2$. If of $D_{i 1} \neq 0$, set $D_{i 2}=0$ and we determine the value $D_{i 1}$ according to a Bernoulli draw with success probability $p_{i 1}^{*}$; if this results in a success $D_{i 1}=1$, otherwise $D_{i 1}=2$.

Conditional on the value of $D$, we proceed to simulate the exposure $X$. If $D_{i j}=0$, we generated a binary exposure $X_{i j}$ with success probability given by $\log i t\left(p_{i j}\right)=\gamma_{0 i}+\gamma_{1} Z_{i j}$. If $D_{i j}=k$ we generate the binary exposure variable with success probability, $\operatorname{logit}\left(p_{i j}\right)=$ $\gamma_{0 i}+\gamma_{1} Z_{i j}+\beta_{2 k}, j, k=1,2$.

We performed two sets of simulations, one with a constant value of $\gamma_{0 i}$ namely -1.00 the other with a relatively varying set of $\gamma_{0 i}$ 's simulated from a normal distribution with mean -0.5 and standard deviation 1.5 . We assumed $N(0,5)$ prior on all the relative risk parameters and a Gamma $(2,2)$ prior for $\alpha$. In all our simulations we used identical parameters for the normal distribution which is assumed to be prior on $\gamma_{0}$ in PBC and the i.i.d. prior on $\gamma_{0 i}$ in PBV and also as the mixing distribution in the Dirichlet Process prior for $\operatorname{BSP}(N(0,6)$ in this case). We replicated the simulation 50 times, generating 50 different datasets and 
obtained the parameter estimates by above mentioned methods and computed their average and MSE. For each replication we also generated data with 30\% exposure values missing completely at random and recalculated all the estimates.

The simulation results presented in Tables 4 and 5 illustrate that for constant stratum effect, the three methods are comparable with PBV estimates being furthest from the true parameters of interest whereas for varying stratum effect the BSP and PBV methods have a clear edge over the PBC method. Overall BSP seems to be the more robust choice as at the onset of a study one is not sure about the nature of variability in the stratum effects.

\section{Conclusion}

In this paper we proposed a semiparametric Bayesian method to analyze matched casecontrol data with more than one disease state and illustrate the methods with a real example. The simulation results indicate that in presence of stratum variability and missing data the Bayesian semiparametric method is superior to the parametric Bayesian alternatives. All three methods perform comparably with constant stratum effects.

Our proposed model considers a non-deterministic exposure variable having a probability distribution belonging to the exponential family. Moreover, the distribution of the exposure could be different in different strata. Our model takes into account both discrete and continuous exposure along with possible missingness in the exposure variable. The growing number of stratum effect parameters are modeled in a semiparametric Bayesian way to overcome the inconsistency problems arising out of classical analysis of such matched data. The computations involving a Dirichlet process prior with a mixing normal distribution are done through a suitable MCMC scheme and enable us to obtain estimates of the parameters of interest. The method could be extended to multiple exposures having an underlying association pattern as well. The general framework is extremely flexible for being used in unorthodox data situations involving missingness and measurement error as well as incorporating widely 
different types of exposure variables that one may come across in practice.

\section{ACKNOWLEDGEMENTS}

The research of Sinha and Ghosh was partially supported by the NIH grant R01-85414. Mukherjee's research was partially supported by the New Researchers' scholarship sponsored through Stanford University. The software needed to carry out the data analysis is available at http://stat.ufl.edu/ mukherjee. We are grateful to the associate editor for his/her valuable comments. 


\section{REFERENCES}

Antoniak, C. E. (1974). Mixtures of Dirichlet processes with applications to non-parametric problems. The Annals of Statistics 2, 1152-1174.

Ashby, D., Hutton, J. L. and McGee, M. A. (1993). Simple Bayesian analyses for casecontrolled studies in cancer epidemiology. Statistician 42, 385-389.

Breslow, N. E. and Day, N. E. (1980). Statistical Methods in Cancer Research, Volume 1. Lyon: International Agency for Research on Cancer.

Escobar, M. D. and West, M. (1995). Bayesian density estimation and inference using mixtures. Journal of the American Statistical Association 90, 577-588.

Gelman, A. and Rubin, D. B. (1992). Inference from iterative simulation using multiple sequences (with discussion). Statistical Science 7, 457-511.

Hosmer, D. A. and Lemeshow, S. (2000). Applied Logistic Regression, 2nd edition. New York: John Wiley.

Little, R. J. A. and Rubin, D. B. (1987). Statistical Analysis with Missing Data. New York: John Wiley.

Müller, P. and Roeder, K. (1997). A Bayesian semiparametric model for case-control studies with errors in variables. Biometrika $\mathbf{8 4}, 523-537$.

Müller, P., Parmigiani, G., Schildkraut, J. and Tardella, L. (1999). A Bayesian Hierarchical Approach for Combining Case-Control and Prospective studies. Biometrics 55, 858-866.

Neal, R. M. (2000). Markov chain sampling methods for Dirichlet process mixture models. Journal of Computational and Graphical Statistics 9, 249-265.

Nurminen, M. and Mutanen, P. (1987). Exact Bayesian analysis of two proportions. Scandinavian Journal of Statistics 14, 67-77.

Paik, M. C. and Sacco, R. (2000). Matched case-control data analyses with missing covariates. Applied Statistics 49, 145-156.

Prentice, R. L. and Pyke, R. (1979). Logistic disease incidence models and case-control studies. Biometrika 66, 403-411.

Rathouz, P. J., Satten, G. A. and Carroll, R. J. (2002). Semiparametric inference in matched case-control studies with missing covariate data. Biometrika 89, 905-916.

Satten, G. A. and Carroll, R. J. (2000). Conditional and unconditional categorical regression models with missing covariates. Biometrics 56, 384-388.

Satten, G. and Kupper, L. (1993a). Conditional regression analysis of the odds ratio between two binary variables when one is not measured with certainty: a method for epidemiologic 
studies. Biometrics 49, 429-440.

Satten, G. A. and Kupper, L. (1993b). Inferences about exposure-disease associations using probability-of-exposure information. Journal of the American Statistical Association 88, 200-208.

Seaman, S. R. and Richardson, S. (2001). Bayesian analysis of case-control studies with categorical covariates. Biometrika 88, 1073-1088.

Sinha, S., Mukherjee, B., Mallick, B. K., Ghosh, M. and Carroll, R. (2003). Semiparametric Bayesian analysis of matched case-control studies with missing exposure. Preprint.

Zelen, M. and Parker, R. A. (1986). Case-control studies and Bayesian inference. Statistics in Medicine 5, 261-269.

\section{APPENDIX}

\section{A1. Full conditional distributions for the parameters:}

The following are the forms of the full conditional distributions of the parameters:

As stated before, here we have assumed the following set priors $\boldsymbol{\beta}_{1 k} \sim N_{2}\left(\boldsymbol{\mu}_{\beta_{1 k}}, \sigma_{\beta_{1 k}}^{2} \boldsymbol{I}\right)$, for $k=1,2, \boldsymbol{\beta}_{2} \sim N_{2}\left(\boldsymbol{\mu}_{\beta_{2}}, \sigma_{\beta_{2}}^{2} \boldsymbol{I}\right)$ and $\gamma_{1} \sim N_{2}\left(\boldsymbol{\mu}_{\gamma_{1}}, \sigma_{\gamma_{1}}^{2} \boldsymbol{I}\right)$.

$$
\pi\left(\boldsymbol{\beta}_{1 k} \mid \cdot\right) \propto \frac{\exp \left[-\frac{1}{2 \sigma_{\beta_{1 k}^{2}}^{2}}\left(\boldsymbol{\beta}_{1 k}-\boldsymbol{\mu}_{\beta_{1 k}}-\sigma_{\beta_{1 k}}^{2} \sum_{i=1}^{n} \boldsymbol{h}_{i} Z_{i 1}^{(k)}\right)^{T}\left(\boldsymbol{\beta}_{1 k}-\boldsymbol{\mu}_{\beta_{1 k}}-\sigma_{\beta_{1 k}}^{2} \sum_{i=1}^{n} \boldsymbol{h}_{i} Z_{i 1}^{(k)}\right)\right]}{\prod_{i=1}^{n}\left\{\sum_{j=1}^{M+1} \exp \left(\boldsymbol{h}_{i}^{T} \boldsymbol{\beta}_{11} Z_{i j}^{(1)}+\boldsymbol{h}_{i}^{T} \boldsymbol{\beta}_{12} Z_{i j}^{(2)}\right) \times \frac{\left(1+\exp \left(\theta_{i j}^{\star}\right)\right)}{\left(1+\exp \left(\theta_{i j}\right)\right)}\right\}}
$$

for $k=1,2$.

$$
\begin{aligned}
& \pi\left(\boldsymbol{\beta}_{2} \mid \cdot\right) \propto \frac{\exp \left[-\frac{1}{2 \sigma_{\beta_{2}}^{2}}\left(\boldsymbol{\beta}_{2}-\boldsymbol{\mu}_{\beta_{2}}-\sigma_{\beta_{2}}^{2} \sum_{i=1}^{n} \delta_{i 1} \boldsymbol{h}_{i} X_{i 1}\right)^{T}\left(\boldsymbol{\beta}_{2}-\boldsymbol{\mu}_{\beta_{2}}-\sigma_{\beta_{2}}^{2} \sum_{i=1}^{n} \delta_{i 1} \boldsymbol{h}_{i} X_{i 1}\right)\right]}{\prod_{i=1}^{n}\left\{\sum_{j=1}^{M+1} \exp \left(\boldsymbol{h}_{i}^{T} \boldsymbol{\beta}_{11} Z_{i j}^{(1)}+\boldsymbol{h}_{i}^{T} \boldsymbol{\beta}_{12} Z_{i j}^{(2)}\right) \times \frac{\left(1+\exp \left(\theta_{i j}^{\star}\right)\right)}{\left(1+\exp \left(\theta_{i j}\right)\right)}\right\}} \\
& \times \prod_{i=1}^{n}\left\{1+\exp \left(\theta_{i 1}^{*}\right)\right\}^{1-\delta_{i 1}} \text {. } \\
& \pi\left(\boldsymbol{\gamma}_{1} \mid \cdot\right) \propto \frac{\exp \left[-\frac{1}{\sigma_{\gamma_{1}}^{2}}\left(\boldsymbol{\gamma}_{1}-\boldsymbol{\mu}_{\gamma_{1}}-\sigma_{\gamma_{1}}^{2} \sum_{i=1}^{n} \sum_{j=1}^{M+1} \delta_{i j} \mathbf{Z}_{i j} X_{i j}\right)^{T}\left(\boldsymbol{\gamma}_{1}-\boldsymbol{\mu}_{\gamma_{1}}-\sigma_{\gamma_{1}}^{2} \sum_{i=1}^{n} \sum_{j=1}^{M+1} \delta_{i j} \mathbf{Z}_{i j} X_{i j}\right)\right]}{\prod_{i=1}^{n}\left\{\sum_{j=1}^{M+1} \exp \left(\boldsymbol{h}_{i}^{T} \boldsymbol{\beta}_{11} Z_{i j}^{(1)}+\boldsymbol{h}_{i}^{T} \boldsymbol{\beta}_{12} Z_{i j}^{(2)}\right) \times \frac{\left(1+\exp \left(\theta_{i j}^{\star}\right)\right)}{\left(1+\exp \left(\theta_{i j}\right)\right)}\right\}} \\
& \times \prod_{i=1}^{n}\left\{\frac{\left(1+\exp \left(\theta_{i 1}^{*}\right)\right)^{1-\delta_{i 1}}}{\left(1+\exp \left(\theta_{i 1}\right)\right)} \prod_{j=2}^{M+1}\left(1+\exp \left(\theta_{i j}\right)\right)^{-\delta_{i j}}\right\} \text {. } \\
& \pi\left(\gamma_{0 i} \mid \cdot\right) \propto \sum_{k \neq i} \frac{1}{\alpha+n-1} w_{k}\left(\boldsymbol{\beta}_{11}, \boldsymbol{\beta}_{12}, \boldsymbol{\beta}_{2}, \boldsymbol{\gamma}_{1}, \gamma_{0 s}, s \neq i\right) I\left(\gamma_{0 k}\right) L_{c} \\
& +w_{i}\left(\boldsymbol{\beta}_{11}, \boldsymbol{\beta}_{12}, \boldsymbol{\beta}_{2}, \boldsymbol{\gamma}_{1}, \gamma_{0 s}, s \neq i\right) f_{i}\left(\gamma_{0 i} \mid \cdot\right) \text {. }
\end{aligned}
$$


where,

$$
\begin{aligned}
f_{i}\left(\gamma_{0 i} \mid \cdot\right) & \propto \frac{\exp \left[-\frac{1}{2 \sigma_{0}^{2}}\left(\gamma_{0 i}-\xi_{0}-\sigma_{0}^{2} \sum_{i=1}^{n} \sum_{j=1}^{M+1} \delta_{i j} X_{i j}\right)^{2}\right]}{\prod_{i=1}^{n}\left\{\sum_{j=1}^{M+1} \exp \left(\boldsymbol{h}_{i}^{T} \boldsymbol{\beta}_{11} Z_{i j}^{(1)}+\boldsymbol{h}_{i}^{T} \boldsymbol{\beta}_{12} Z_{i j}^{(2)}\right) \times \frac{\left(1+\exp \left(\theta_{i j}^{\star}\right)\right)}{\left(1+\exp \left(\theta_{i j}\right)\right)}\right\}} \\
& \times \prod_{i=1}^{n}\left\{\frac{\left(1+\exp \left(\theta_{i 1}^{*}\right)\right)^{1-\delta_{i 1}}}{\left(1+\exp \left(\theta_{i 1}\right)\right)} \prod_{j=2}^{M+1}\left(1+\exp \left(\theta_{i j}\right)\right)^{-\delta_{i j}}\right\} .
\end{aligned}
$$

and $w_{r}, r=1, \cdots, n$ are weight functions such that $\pi\left(\gamma_{0 i} \mid \cdot\right)$ is a proper density. One may note that if $X$ is completely observed $\delta_{i j}=1 \forall i, j$.

Remark 3. For resampling from the full conditional distribution of $\alpha$ we followed the algorithm suggested by Escobar and West (1995). At each step we counted the number of distinct $\gamma_{0 i}$, say $k$, and conditional on the current values of $\alpha$ and $k$, we simulated a latent beta random variable say, $\eta \sim B(\alpha+1, n)$. Let $G(a, b)$ denote the prior on $\alpha$. Using the current value of $k$ and $\eta, \alpha$ was simulated from the following mixture of Gamma distribution,

$$
\pi(\alpha \mid \eta, k)=p G(a+k, b-\log (\eta))+(1-p) G(a+k-1, b-\log (\eta))
$$

where $p=(a+k-1) /[a+k-1+n\{b-\log (\eta)\}]$. 


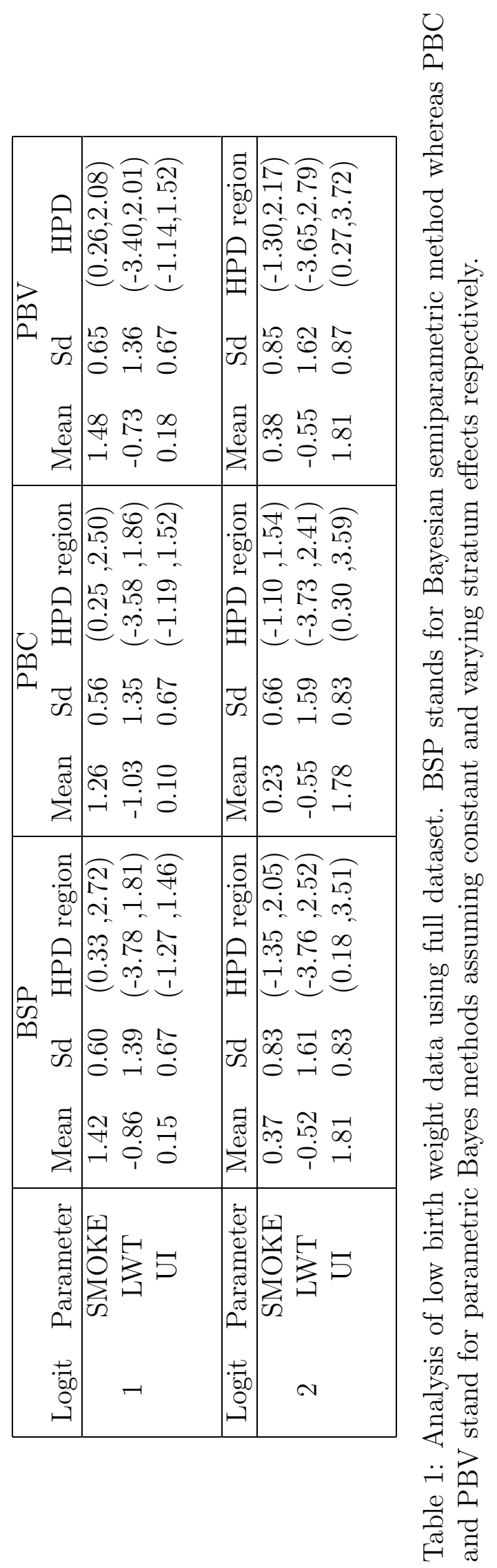




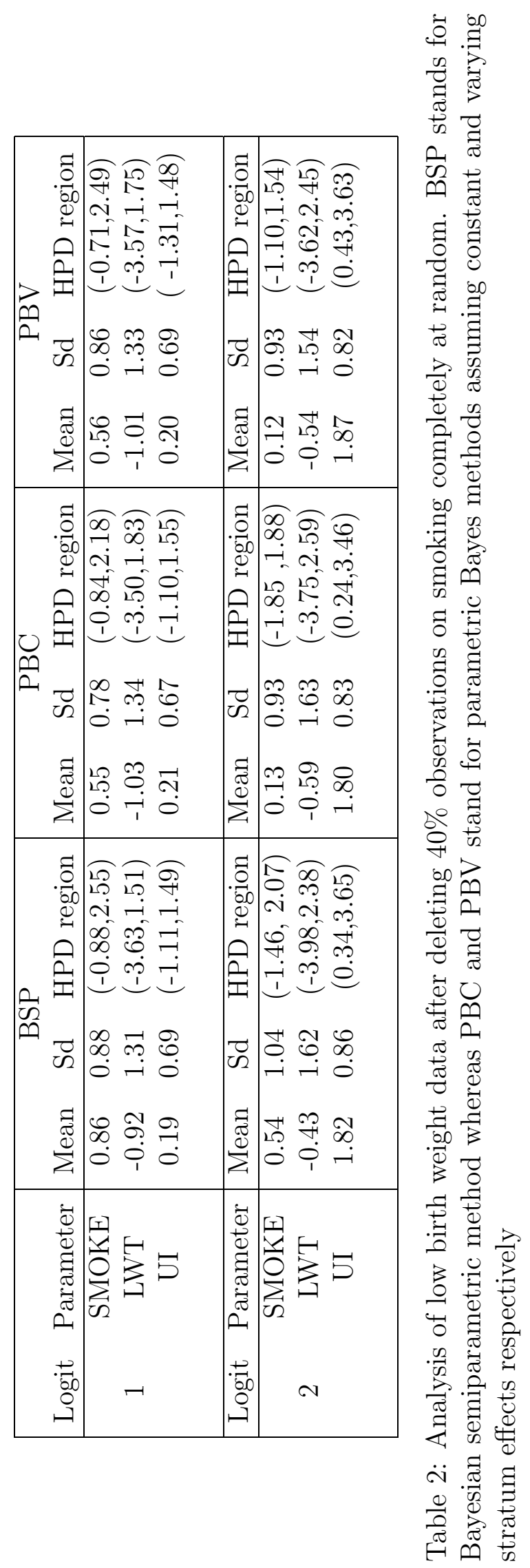




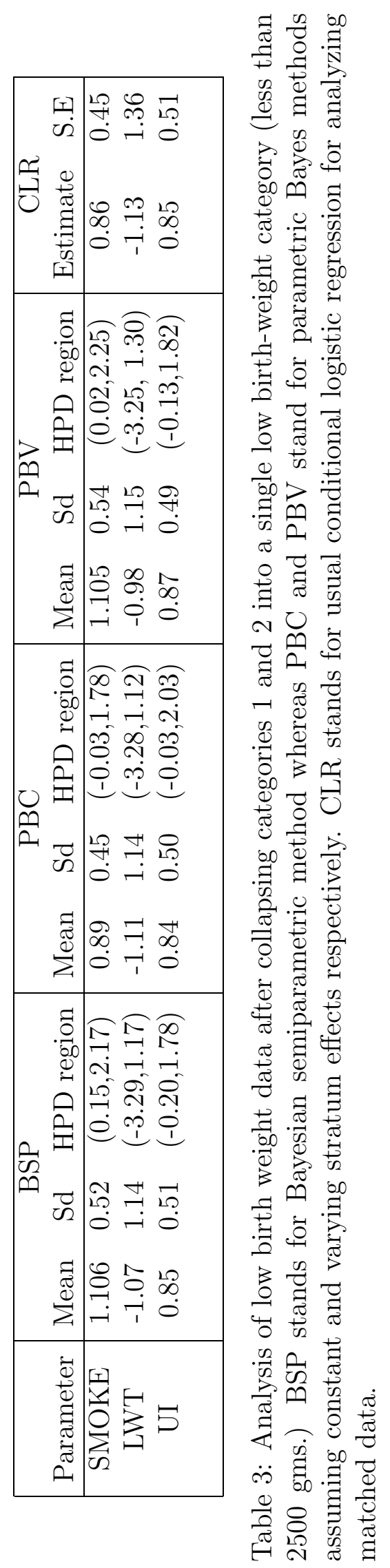




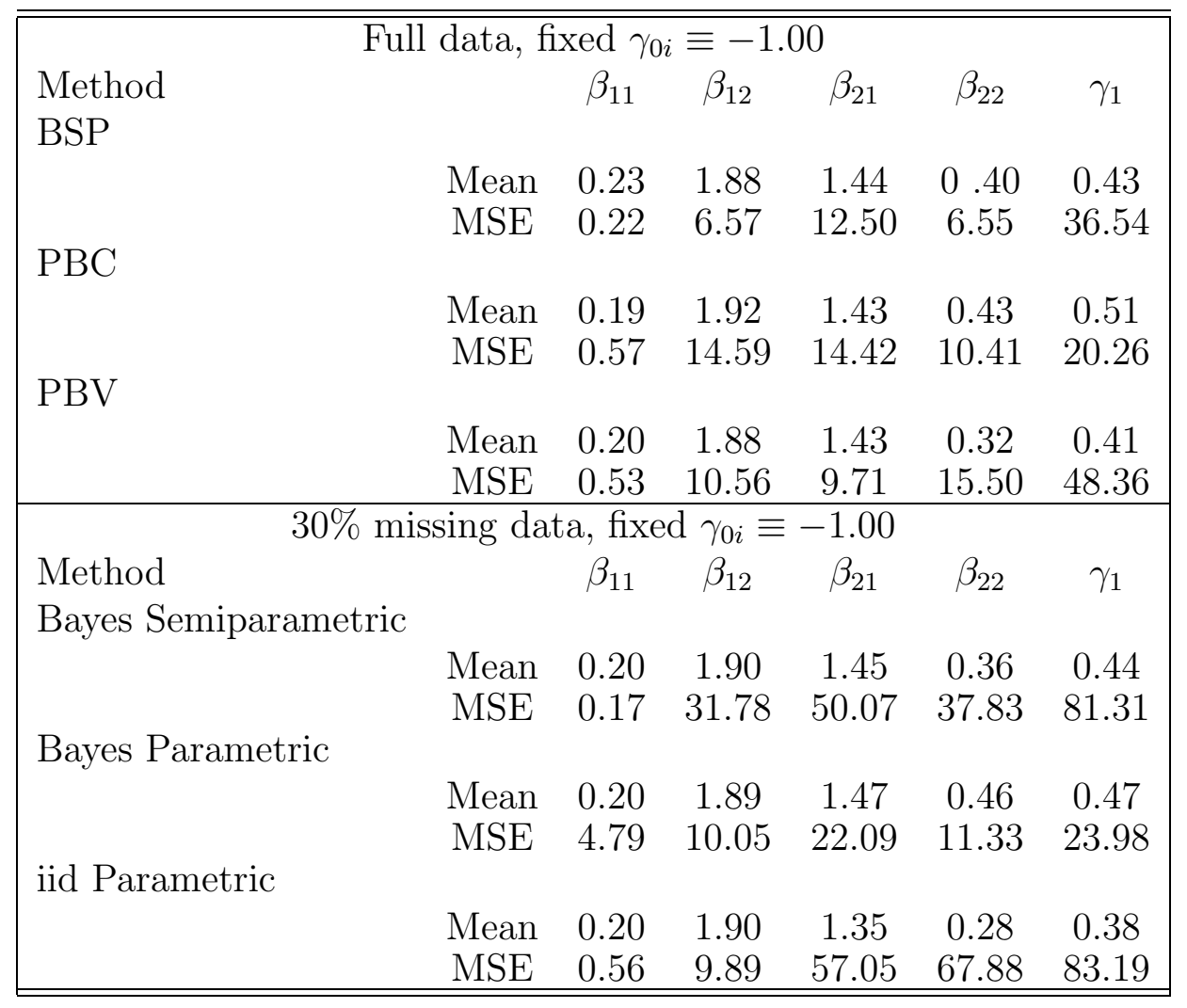

Table 4: Results of the simulation study. Here "Mean" is the simulated mean, while MSE is the mean squared error $\times 1000$. The true parameter values are $\beta_{11}=0.20, \beta_{12}=1.80$, $\beta_{21}=1.40, \beta_{22}=0.40$ and $\gamma_{1}=0.60$. BSP stands for Bayesian semiparametric method whereas PBC and PBV stand for parametric Bayes methods assuming constant and varying stratum effects respectively. 


\begin{tabular}{|c|c|c|c|c|c|c|}
\hline \multicolumn{7}{|c|}{ Full data, varying $\gamma_{0 i} \sim N(-0.5,2.25)$} \\
\hline \multirow{2}{*}{$\begin{array}{l}\text { Method } \\
\text { BSP }\end{array}$} & & $\beta_{11}$ & $\beta_{12}$ & $\beta_{21}$ & $\beta_{22}$ & $\gamma_{1}$ \\
\hline & Mean & 0.19 & 1.89 & 1.43 & 0.42 & 0.50 \\
\hline \multicolumn{7}{|l|}{$\mathrm{PBC}$} \\
\hline & Mean & 0.20 & 1.89 & 1.52 & 0.48 & 0.53 \\
\hline \multirow{3}{*}{ PBV } & MSE & 0.70 & 8.48 & 27.98 & 13.36 & 97.75 \\
\hline & Mean & 0.19 & 1.92 & 1.44 & 0.43 & 0.51 \\
\hline & MSE & 0.65 & 12.84 & 11.15 & 9.07 & 13.25 \\
\hline \multicolumn{7}{|c|}{$30 \%$ missing data, varying $\gamma_{0 i} \sim N(-0.5,2.25)$} \\
\hline \multirow{3}{*}{$\begin{array}{l}\text { Method } \\
\text { BSP }\end{array}$} & & $\beta_{11}$ & $\beta_{12}$ & $\beta_{21}$ & $\beta_{22}$ & $\gamma_{1}$ \\
\hline & Mean & 0.20 & 1.91 & 1.50 & 0.44 & 0.50 \\
\hline & \multicolumn{6}{|c|}{$\mathrm{PBC}$} \\
\hline \multirow{4}{*}{ PBV } & Mean & 0.20 & 1.92 & 1.54 & 0.49 & 0.53 \\
\hline & MSE & 0.46 & 14.04 & 30.28 & 14.68 & 15.54 \\
\hline & Mean & 0.20 & 1.94 & 1.49 & 0.44 & 0.50 \\
\hline & MSE & 0.76 & 14.31 & 16.15 & 14.42 & 19.35 \\
\hline
\end{tabular}

Table 5: Results of the simulation study. Here "Mean" is the simulated mean, while MSE is the mean squared error $\times 1000$. The true parameter values are $\beta_{11}=0.20, \beta_{12}=1.80$, $\beta_{21}=1.40, \beta_{22}=0.40$ and $\gamma_{1}=0.60$. BSP stands for Bayesian semiparametric method whereas PBC and PBV stand for parametric Bayes methods assuming constant and varying stratum effects respectively. 


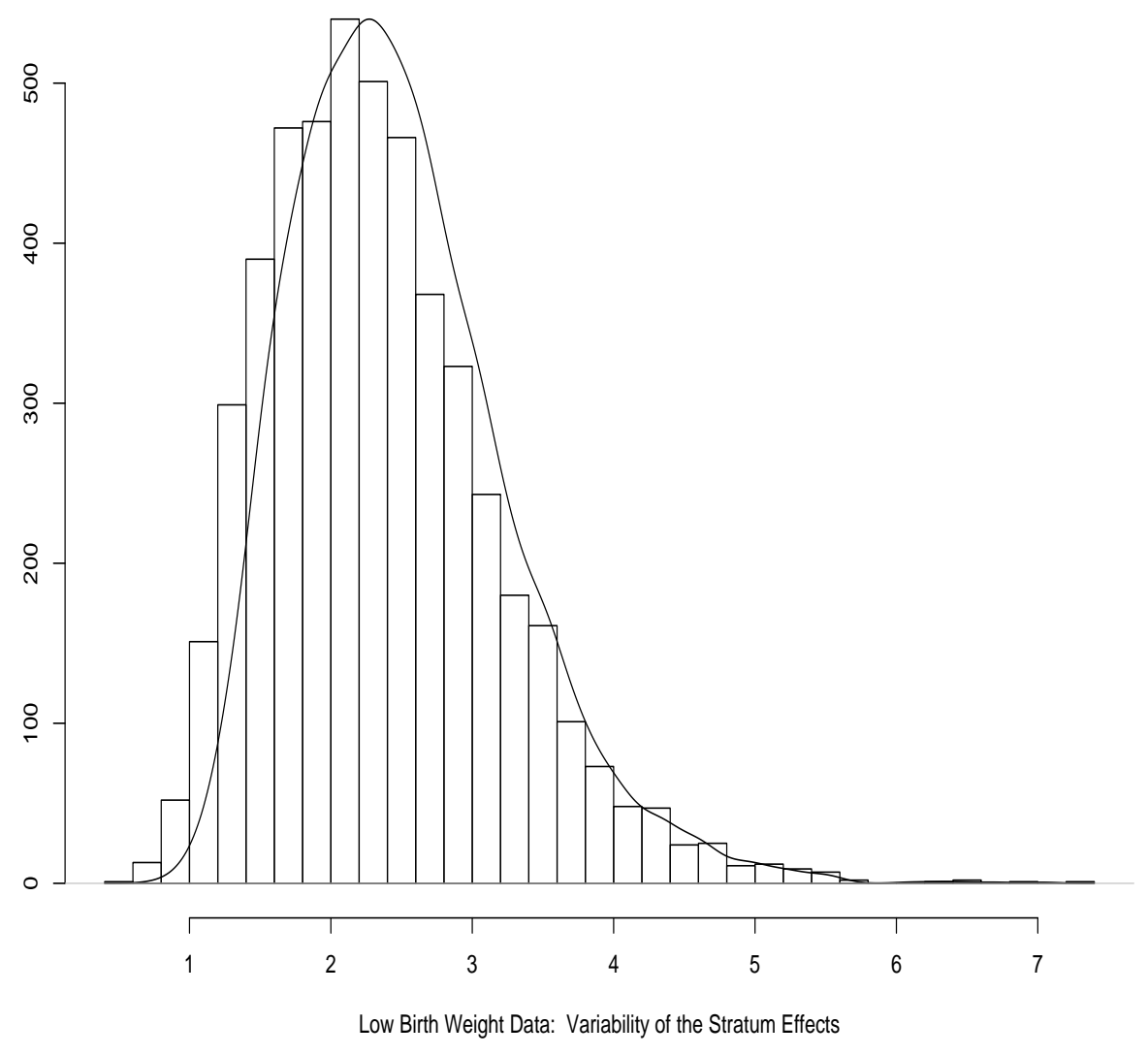

Figure 1: Plot of the variability of $\gamma_{0 i}$ 's for the Low Birthweight study example. Estimates of the $29 \gamma_{0 i}$ 's were collected for each of the last 3000 MCMC samples. Variances of these 29 values were then calculated for each run. The histogram is of these 3000 variance values with a kernel density estimate overlayed on it. 